\title{
Generalized Linear-Phase Lapped Orthogonal Transforms
}

\author{
R. L. de Queiroz $z^{1 *}$ T. Q. Nguyen ${ }^{2}$ and K.R.Rao \\ ${ }^{1,3}$ Electrical Engineering Department University of Texas at Arlington \\ Box 19016, Arlington, TX, $76019^{1}$ queiroz@eepost.uta.edu ${ }^{3}$ eekrr521@utacnvx.uta.edu \\ ${ }^{2}$ MIT Lincoln Laboratory, Rm. D-361 \\ 244 Wood St., Lexington, MA 02173-9108 nguyen@ll.mit.edu
}

\begin{abstract}
The general factorization of a linear-phase paraunitary filter bank (LPPUFB) is revisited and we introduce a class of lapped orthogonal transforms with extended overlap (GenLOT). In this formulation, the discrete cosine transform (DCT) is the order-1 GenLOT, the lapped orthogonal transform is the order-2 GenLOT, and so on, for any filter length which is an integer multiple of the block size. All GenLOTs are based on the DCT and have fast implementation algorithms. The degrees of freedom in the design of GenLOTs are described and design examples are presented along with some practical applications.
\end{abstract}

\section{Introduction}

The discrete cosine transform (DCT) [1] is used in most of the international standards for image compression and for several signal processing tasks. However, the DCT sometimes leads to discontinuities across the block boundaries after the processed signal is inversed transformed [1]. Recently the lapped orthogonal transform (LOT) [2] was developed as a competitive alternative because of its extended basis functions which overlap across traditional block boundaries, thus eliminating the blocking effect. Also, the LOT has good performance and possesses a fast implementation algorithm based on the DCT for which several algorithms, VLSI chips, and computer programs have been developed [1]. It is well known that the DCT and the LOT are particular choices of FIR linear-phase paraunitary filter banks (LPPUFB) $[3,6]$. Linear-phase filter banks have been studied extensively and several design approaches can be found in the literature (see, for example, [3-5]). However, fast implementation algorithms were usually ignored. Very recently, a minimal structure to implement all LPPUFB (where the filters' lengths are the same) was developed $[7,8]$. We will introduce a particular simplification leading to a class of LPPUFB which we call the generalized linear-

${ }^{*}$ This work was supported in part by CNPq, Brazil, under Grant 200.804-90-1. phase lapped orthogonal transforms (GenLOTs). The GenLOTs have a fast implementation algorithm based on the DCT, and both DCT and LOT can be regarded as special cases.

In terms of notation, the following conventions are adopted: $\mathbf{I}_{n}: n \times n$ identity matrix. $\mathbf{J}_{n}: n \times n$ counteridentity or reversing matrix. $\mathbf{0}_{n}: n \times n$ null matrix. ()$^{R}$ : column and row reversal of a matrix. For example, if $\mathbf{A}$ is a $n \times n$ matrix, then $\mathbf{A}^{R}=\mathbf{J}_{n} \mathbf{A} \mathbf{J}_{n}$.

\section{LOT}

The DCT is implemented by segmenting the input signal into blocks of $M$ samples and transforming each one independently. The LOT allows overlap of the basis functions. As an example for $M=8$, the implementation algorithm for the LOT is shown in Fig. 1. It is wellknown [6] that the LOT is a $M$-channel uniform FIR filter bank, where the filters have length $L=2 M$ and their coefficients are formed by the coefficients of the basis functions. Hence, as the basis functions are symmetric, the LOT can be regarded as a linear-phase filter bank. It is also easy to show that the corresponding filter bank is also paraunitary, so that the LOT is a particular LPPUFB [6].

\section{Generalized LOT}

Consider a uniform maximally-decimated $M$-channel FIR filter bank for which we impose some restrictions. First, we assume that $M$, the number of channels, is even and that the filters have linear-phase. Second, we assume the filters have length $L$ which is an integer multiple of $M$ as $L=N M$. Third, the filter bank is assumed to be paraunitary. Hence $[3,6]$, we have $g_{i}(n)=f_{i}(L-1-n)$, for $0 \leq i \leq M-1$ and $0 \leq n \leq L-1$. Also, from [7,8], we know that $M / 2$ filters (in analysis or synthesis) have symmetric impulse responses and the other $M / 2$ filters have anti-symmetric impulse responses.

$$
g_{i}(n)=f_{i}(L-1-n)= \pm f_{i}(n) .
$$


Alternatively, we can develop the filter bank by segmenting the signal into blocks of $M$ samples. Let the input signal $x(n)$ be expressed by its $M$ polyphase components $x_{i}(m)[3]$, as $x_{i}(m)=x(m M+i)$ where $0 \leq i \leq M-1$. For a given instant $m$ the $M$ polyphase samples form the $m$-th block of $M$ samples. The subband signals $y_{k}(m)$ are directly related to the polyphase components by a multi-input multi-output (MIMO) discrete transfer matrix with FIR filter entries [3] known as the polyphase transfer matrix (PTM). In a PR-LPPUFB, for analysis and synthesis, the MIMO PTM is given by $\mathbf{E}(z)$ and $\tilde{\mathbf{E}}(z)=z^{-(N-1)} \mathbf{E}^{T}\left(z^{-1}\right)$, respectively. Under the assumptions on $L, M$, and on the filter symmetry, we know that $[7,8] \mathbf{E}(z)$ for the LPPUFB of degree $N-1$ can be decomposed as a product of orthogonal factors and delays as

$$
\mathbf{E}(z)=\mathbf{S Q T}_{N-1} \Lambda(z) \mathbf{T}_{N-2} \Lambda(z) \cdots \Lambda(z) \mathbf{T}_{0} \mathbf{Q}
$$

where

$$
\begin{gathered}
\mathbf{Q}=\left[\begin{array}{ll}
\mathbf{I}_{M / 2} & \mathbf{0}_{M / 2} \\
\mathbf{0}_{M / 2} & \mathbf{J}_{M / 2}
\end{array}\right] \\
\Lambda(z)=\left[\begin{array}{rr}
\mathbf{I}_{M / 2} & \mathbf{0}_{M / 2} \\
\mathbf{0}_{M / 2} & z^{-1} \mathbf{I}_{M / 2}
\end{array}\right] \\
\mathbf{S}=\frac{1}{\sqrt{2}}\left[\begin{array}{lr}
\mathbf{S}_{0} & \mathbf{0}_{M / 2} \\
\mathbf{0}_{M / 2} & \mathbf{S}_{1}
\end{array}\right]\left[\begin{array}{rr}
\mathbf{I}_{M / 2} & \mathbf{J}_{M / 2} \\
\mathbf{I}_{M / 2} & -\mathbf{J}_{M / 2}
\end{array}\right],
\end{gathered}
$$

$\mathbf{S}_{0}$ and $\mathbf{S}_{1}$ can be any $M / 2 \times M / 2$ orthogonal matrices, and $\mathbf{T}_{i}$ are $M \times M$ orthogonal matrices described as

$$
\mathbf{T}_{i}=\left[\begin{array}{ll}
\mathbf{A}_{i} & \mathbf{B}_{i} \\
\mathbf{B}_{i} & \mathbf{A}_{i}
\end{array}\right]
$$

We will abbreviate the notation for (2) as

$$
\mathbf{E}(z)=\mathbf{S Q T}_{N-1}\left(\prod_{i=N-2}^{0} \Lambda(z) \mathbf{T}_{i}\right) \mathbf{Q} .
$$

Let

$$
\mathbf{W}=\frac{1}{\sqrt{2}}\left[\begin{array}{cc}
\mathbf{I}_{M / 2} & \mathbf{I}_{M / 2} \\
\mathbf{I}_{M / 2} & -\mathbf{I}_{M / 2}
\end{array}\right] \quad \Phi_{i}=\left[\begin{array}{cc}
\mathbf{U}_{i} & \mathbf{0}_{M / 2} \\
\mathbf{0}_{M / 2} & \mathbf{V}_{i}
\end{array}\right]
$$

where $\mathbf{U}_{i}$ and $\mathrm{V}_{i}$ can be any $M / 2 \times M / 2$ orthogonal matrices. Note that $\mathbf{T}_{i}$ can be expressed as $[7,8]$

$$
\mathbf{T}_{i}=\mathbf{W} \Phi_{i} \mathbf{W}
$$

for $\mathbf{A}_{i}=\left(\mathbf{U}_{i}+\mathbf{V}_{i}\right) / 2$ and $\mathbf{B}_{i}=\left(\mathbf{U}_{i}-\mathbf{V}_{i}\right) / 2$.

Then, it is easy to see that $\mathbf{S Q T} \mathbf{T}_{N-1}$ can be simplified to

$$
\mathbf{S Q T}_{N-1}=\left[\begin{array}{cc}
\mathbf{S}_{0} \mathbf{U}_{N-1} & \mathbf{0}_{M / 2} \\
\mathbf{0}_{M / 2} & \mathbf{S}_{1} \mathbf{V}_{N-1}
\end{array}\right] \mathbf{W}
$$

As $\mathbf{U}_{N-1}$ and $\mathbf{S}_{0}$ are generic orthogonal matrices, and the product $\mathbf{S}_{0} \mathbf{U}_{N-1}$ is also a generic orthogonal matrix, we can discard the term $\mathbf{S}_{0}$ without any loss of generality. The same is valid for $S_{1}$ with regard to $V_{N-1}$. Therefore, we get $\mathbf{S Q T} \mathbf{T}_{N-1}=\boldsymbol{\Phi}_{N-1} \mathbf{W}$ and (7) reduces to

$$
\mathbf{E}(z)=\left(\prod_{i=N-1}^{1} \Phi_{i} \mathbf{W} \Lambda(z) \mathbf{W}\right) \mathbf{E}_{0}
$$

where $\mathbf{E}_{0}=\Phi_{0} \mathbf{W Q}$ is a general $M \times M$ orthogonal matrix with symmetric basis functions, i.e., the PTM of order 0 of a LPPUFB. Since an order- $n$ PTM leads to filters of length $(n+1) M$, a LPPUFB with filter length $n M+M$ can be obtained from one with filter length $n M$ by adding a stage to the PTM of the latter. If $\mathbf{E}_{n}(z)$ denotes an order- $n$ PTM, then we can state that

$$
\mathbf{E}_{n}(z)=\mathbf{K}_{n}(z) \mathbf{E}_{n-1}(z)
$$

where

$$
\mathbf{K}_{i}(z)=\Phi_{i} \mathbf{W} \Lambda(z) \mathbf{W} .
$$

Therefore, for any $N>1$, any PTM of a LPPUFB can be expressed as

$$
\mathbf{E}(z)=\mathbf{K}_{N-1}(z) \mathbf{K}_{N-2}(z) \cdots \mathbf{K}_{1}(z) \mathbf{E}_{0}
$$

As a remark, it can be shown that the stage $\mathbf{K}_{i}(z)$ can be expressed as

$$
\mathbf{K}_{i}(z)=\Phi_{i} \mathbf{W}_{1} \Lambda(z) \mathbf{W}_{2}
$$

where $\mathbf{W}_{1}$ and $\mathbf{W}_{2}$ can be either $\mathbf{W}$ or $\mathbf{W}^{R}$, independently.

The GenLOT is defined as a LPPUFB obeying (14), where $\mathbf{E}_{0}$ is chosen to be the DCT matrix [1], which we denote as D. The output of the DCT is, then, separated into groups of even and odd coefficients. The GenLOT with $N-1$ stages after the DCT has basis functions (filters) with length $L=N M$ and has its PTM defined as

$$
\mathbf{E}(z)=\mathbf{K}_{N-1}(z) \mathbf{K}_{N-2}(z) \cdots \mathbf{K}_{1}(z) \mathbf{D} .
$$

The implementation flow-graphs for the analysis and synthesis sections are shown in Fig. 2. In this figure, each branch carries $M / 2$ samples.

The class of GenLOTs, defined in this way, allows us to view the DCT and LOT as special cases, respectively for $N=1$ and $N=2$. The degrees of freedom reside on the matrices $\mathbf{U}_{i}$ and $\mathbf{V}_{i}$ which are only restricted to be real $M / 2 \times M / 2$ orthogonal matrices. Thus, each one can be parameterized into a set of $M(M-2) / 8$ plane rotations, (or less, for constrained solutions). 


\section{Design Example}

The LOT can be obtained from the DCT, by direct determination of $\Phi_{1}$ [6]. In this case, $\mathbf{U}_{1}$ and $\mathbf{V}_{1}$ are determined in a general form, without obeying any particular structure. Optimization, in this case, is carried solely to determine an approximation to the matrices $\mathbf{U}_{1}$ and $\mathbf{V}_{1}$, in order to find a faster implementation algorithm [6] However, for $N>2$, there are no techniques available to find all matrices $\Phi_{i}$ directly. The design of a GenLOT is the determination of the free parameters which are the angles for the plane rotations. The solution space is searched through optimization routines, in such a way as to minimize a particular cost function. However, due to the highly non-linear relationships among the angles and the cost functions, there is no guarantee to obtain a global minimum. All GenLOT examples presented here were obtained using unconstrained non-linear optimization and simplex search, using the routines provided by MATLAB version 4.0 .

Features that can be exploited in the design of GenLOT are the transform coding gain $\left(G_{T C}\right)$ [9], the attenuation in the stopband region of each filter, or a combination of both. Other features can be considered as well. Thus, the cost function can be selected as the inverse of any of these functions. In our example we will use examples designed for maximum $G_{T C}$, assuming the input signal as a zero mean $\mathrm{AR}(1)$ signal with adjacent sample correlation coefficient 0.95 (i.e., its autocorrelation function is $r_{x}(n)=0.95^{|n|}$ ).

The basis functions of a GenLOT for $M=8$, optimized for maximum $G_{T C}$ are shown in Fig. 3, for $N=4$ $(L=32)$ and $N=5(L=40)$. We tested the performance of the GenLOT using the maximum $G_{T C}$ design and AR(1) signal model in image coding. The coder algorithm used is the JPEG baseline system [10], merely replacing the $8 \times 8$ DCT by a $8 \times 8$ GenLOT $(N>1)$, obtained through separable implementation of the one-dimensional transform, as is the case for the twodimensional DCT. For the $8 \mathrm{bit} /$ pel $256 \times 256$-pels image "Lena" we tested the JPEG coder comparing the GenLOTs with $N=1$ (DCT), $N=2$ (optimal LOT $[2,6]$ ), $N=4$, and $N=5$, for bit-rates ranging from 0.3 through $1.0 \mathrm{bit} / \mathrm{pel}$. The difference in signal-to-noise ratio (in $\mathrm{dB}$ ) among the GenLOTs and the DCT is shown in Fig. 4.

\section{Conclusions}

The general factorization of LPPUFBs is revisited leading to a new perspective from which the GenLOTs emerged as a trivial particularization. One of the most interesting properties is that the procedure to increase the overlap (filter length) is identical for any order $n$, by applying a post-processing stage $\mathbf{K}_{n}(z)$. The elegance of the factorization and the fact that it is a linear-phase filter bank with a fast algorithm based on the DCT are important attributes for GenLOTs.

The large number of degrees of freedom forced us to use non-linear optimization procedures in the design of GenLOTs. This is not very desirable because we cannot guarantee a global minimum of the cost function, but only a local one. However, for most of our tests, several different initializations led to the same resulting angles, even when very distant starting points were used. This leads us to believe that the optimized solutions are reasonably stable. Further research will concentrate on design issues aimed at specific applications.

\section{References}

[1] K. R. Rao and P. Yip, Discrete Cosine Transform $\therefore$ Algorithms, Advantages, Applications. San Diego, CA : Academic Press, 1990.

[2] H. S. Malvar and D. H. Staelin, "The LOT: transform coding without blocking effects," IEEE Trans. Acoust., Speech, Signal Processing, ASSP-37, pp. 553-559, Apr. 1989.

[3] P.P. Vaidyanathan, Multirate Systems and Filter Banks. Englewood Cliffs, NJ: Prentice-Hall, 1993.

[4] T. Q. Nguyen and P. P. Vaidyanathan, "Structures for M-channel perfect reconstruction FIR QMF banks which yield linear phase analysis filters," IEEE Trans. Acoust., Speech, Signal Processing, vol. 38, pp. 433-446, March 1990.

[5] M. Vetterli and D. Le Gall, "Perfect reconstruction filter banks : some properties and factorizations", IEEE Trans. Acoust., Speech, Signal Processing, ASSP-37, pp. 1057-1071, July 1989.

[6] H. S. Malvar, Signal Processing with Lapped Transforms. Norwood, MA: Artech House, 1992.

[7] A. K. Soman, P. P. Vaidyanathan, and T. Q. Nguyen, "Linear-Phase Orthonormal Filter Banks," Proc. of IEEE Intl. Conf. on Acoust., Speech, Signal Processing, Minneapolis, MN, Vol. III, pp. 209-212, Apr. 1993.

[8] A. K. Soman, P. P. Vaidyanathan, and T. Q. Nguyen, "Linear-phase paraunitary filter banks: theory, factorizations and applications," IEEE Trans, on Signal Processing, vol. 41, Dec. 1993.

[9] N. S. Jayant and P. Noll, Digital Coding of Waveforms. Englewood Cliffs, NJ: Prentice-Hall, 1984.

[10] W. B. Pennebaker and J. L. Mitchell, JPEG: Still Image Compression Standard," New York, NY: Van Nostrand Reinhold, 1993. 


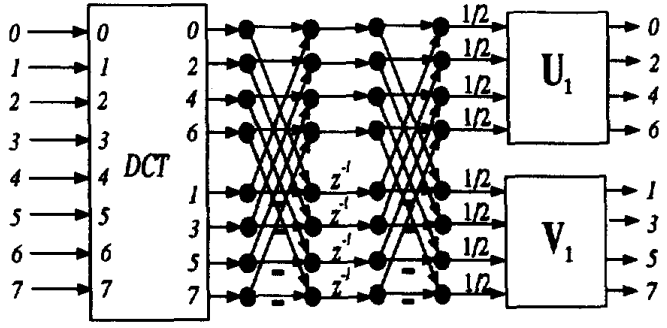

Figure 1. Flow-graph for implementation of the LOT, with blocks of $M=8$ samples.
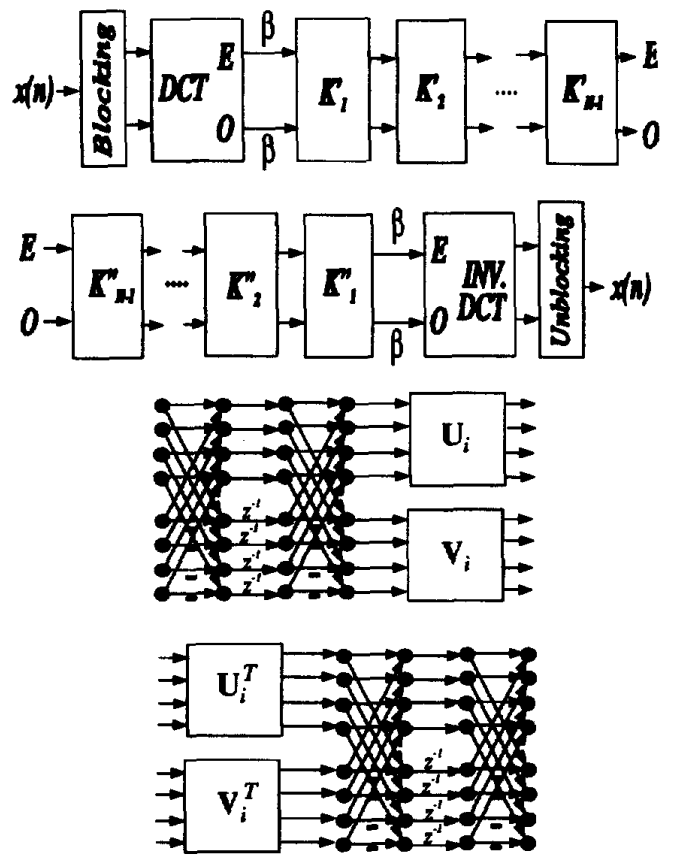

Figure 2. Flow-graph for implementation of GenLOTs. Each branch carries $M / 2$ samples and $E$ and $O$ stand for the even and odd transform coefficents, respectively, of output (analysis) and input (synthesis) for both DCT and GenLOT. Even and odd coefficients also correspond to symmetric and anti-symmetric basis functions (which are the filters' impulse responses), respectively. $\beta$ is a scaling factor incorporating all scaling factors present in $W$, so that $\beta=2^{-(N-1)}$. From top to bottom, we have the diagrams for analysis, synthesis, analysis stage $K_{i}^{\prime}$ (for $M=8$ ), and synthesis stage $K_{i}^{\prime \prime}$ (for $M=8$ ).
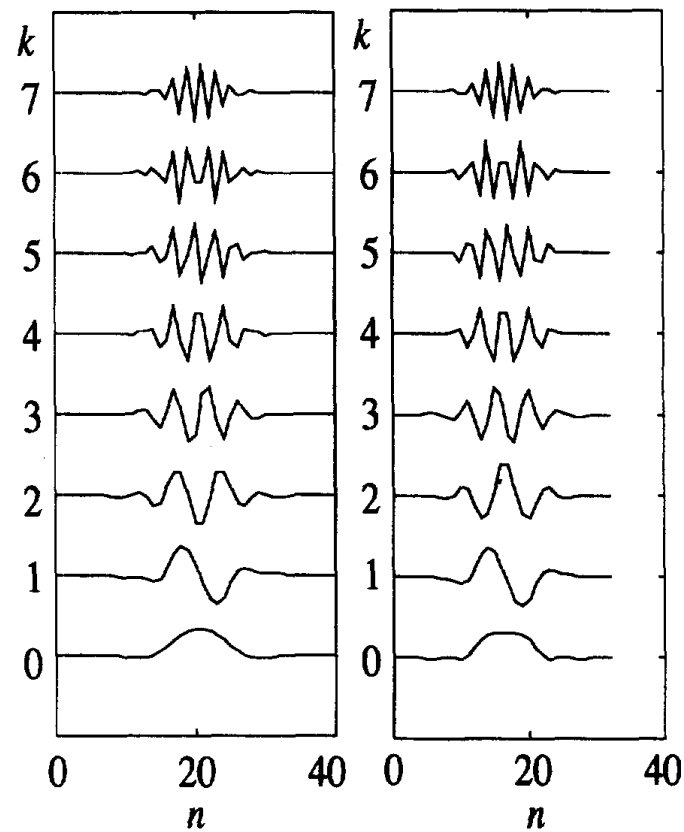

Figure 3. Basis functions $f_{k}(n)$ (filters' impulse responses) of a GENLOT with $M=8$ designed for maximum $G_{T C}$. Examples for $L=40$ (left) and $L=32$ (right) are shown.

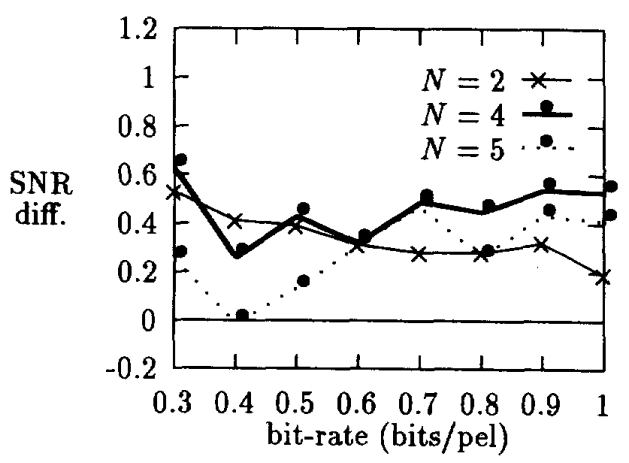

Figure 4. SNR (in $\mathrm{dB}$ ) difference among GenLOTs and the DCT for several bit-rates using test image "Lena". 